\title{
Numerical analysis of global ionospheric current system including the effect of equatorial enhancement
}

\author{
S. Tsunomura \\ Kakioka Magnetic Observatory, Kakioka 595, Yasato, Niihari, Ibaraki, 315-0116, Japan
}

Received: 23 March 1998 / Revised: 19 October 1998 / Accepted: 21 October 1998

\begin{abstract}
A modeling method is proposed to derive a two-dimensional ionospheric layer conductivity, which is appropriate to obtain a realistic solution of the polar-originating ionospheric current system including equatorial enhancement. The model can be obtained by modifying the conventional, thin shell conductivity model. It is shown that the modification for one of the non-diagonal terms $\left(\Sigma_{\theta \varphi}\right)$ in the conductivity tensor near the equatorial region is very important; the term influences the profile of the ionospheric electric field around the equator drastically. The proposed model can reproduce well the results representing the observed electric and magnetic field signatures of geomagnetic sudden commencement. The new model is applied to two factors concerning polar-originating ionospheric current systems. First, the latitudinal profile of the DP2 amplitude in the daytime is examined, changing the canceling rate for the dawnto-dusk electric field by the region 2 field-aligned current. It is shown that the equatorial enhancement would not appear when the ratio of the total amount of the region 2 field-aligned current to that of region 1 exceeds 0.5 . Second, the north-south asymmetry of the magnetic fields in the summer solstice condition of the ionospheric conductivity is examined by calculating the global ionospheric current system covering both hemispheres simultaneously. It is shown that the positive relationship between the magnitudes of high latitude magnetic fields and the conductivity is clearly seen if a voltage generator is given as the source, while the relationship is vague or even reversed for a current generator. The new model, based on the International Reference Ionosphere (IRI) model, can be applied to further investigations in the quantitative analysis of the magnetosphere-ionosphere coupling problems.
\end{abstract}

Key words. Ionosphere (electric fields and currents; equatorial ionosphere; ionosphere-magnetosphere interactions).

\section{Introduction}

Global-scale ionospheric current systems, the driving sources of which are located in the polar region appear in various geomagnetic disturbances, such as storm sudden commencement (SSC), sudden impulse (SI), DP2-type magnetic variation and others. The current systems are categorized into two kinds, DP1 and DP2, according to spatial extent and location. The current systems of SSC and SI are classified in the latter. The DP2-type current system has a wider spatial extension than that of the DP1-type and extends to the equatorial region through middle and low latitudes.

Since geomagnetic phenomena are usually expected to be symmetric or anti-symmetric with respect to the geomagnetic equator, the geomagnetic equator is treated as a boundary of a hemisphere for the global-scale ionospheric current systems. The term 'geomagnetic equator' will be abbreviated to 'equator' hereafter. The boundary condition at the equator is characterized by its high conductance in the very narrow region around the dayside equator. The polar-originating ionospheric current, whose intensity decreases with decreasing latitude from high latitudes, grows very large close to the equator. There have been many investigations of the equatorial enhancement features of sudden commencement (e.g., Araki, 1994; Rastogi, 1993 and references therein) and DP2 (e.g., Nishida et al., 1966; Kikuchi et al., 1996 and references therein). Many articles also discuss the equatorial electric field variation due to magnetospheric processes (e.g., Fejer, 1986, 1991, 1997; Rastogi, 1997; Sastri et al., 1997 and references therein).

Among many kinds of geomagnetic disturbance, the sudden commencement is a good phenomenon to discuss the equatorial enhancement of the polar-originating ionospheric current systems because of its clear variation form and causal relationship. In this work, the term 'SC' will be used to denote the SSC and/or SI 
because the physical mechanisms to cause them are essentially the same. After examinations of the global distribution of the magnetic variation of SC, Araki (1977, 1994) proposed a systematic model of SC decomposing the SC field to the DL, $\mathrm{DP}_{\mathrm{PRI}}$ and $\mathrm{DP}_{\mathrm{MI}}$ fields. Kikuchi and Araki (1979) showed that the SC-associated large-scale horizontal electric field (DP ${ }_{\text {PRI }}$ or $\mathrm{DP}_{\mathrm{MI}}$ field) in the polar ionosphere penetrates instantaneously, by the transmission process of the zeroth order, the TM mode of electromagnetic waves in the wave-guide between the Earth and the ionosphere.

On the basis of the model of Araki (1977), Tsunomura and Araki (1984) made a numerical analysis of the two-dimensional ionospheric current system for $\mathrm{SC}$ and showed that the polar-originating ionospheric current is enhanced in the dayside equator. They obtained a nearly realistic solution for the global distribution of the SC-associated polar-originating ionospheric current. However, recent HF Doppler observations by Sastri et al. (1993) during SCs show different local time dependences of the electric field from the results of Tsunomura and Araki (1984). Tsunomura and Araki's (1984) model should be improved to compare with new observational data.

There have been some simulation studies discussing the ionospheric current system concerning magnetospheric substorm (e.g., Kamide and Matsushita, 1979a, b; Nopper and Carovillano, 1978; Senior and Blanc, 1984; Denisenko and Zamay, 1992). However, these models have some limitations in the treatment of equatorial enhancement of ionospheric conductivity or need a sophisticated numerical technique. The Rice convection model can treat the time-dependent magnetosphere-ionosphere coupling problem comprehensively but neglects or takes a simple assumption for the equatorial enhancement (Harel et al., 1981a, b; Spiro et al., 1988). In this study, a modeling method to obtain the two-dimensional ionospheric conductivity will be proposed as the first improvement of Tsunomura and Araki's (1984) model (Sect. 2.1-2.3). The calculated electric and magnetic fields on the basis of the new model are compared with other models and the observational results for SC (Sect. 3.1).

The versatility of the numerical model, such as the variability in the conductivity distribution, may be useful for the quantitative investigation of the magnetosphereionosphere coupling problems. As a trial for this purpose, the model will be applied to the two matters concerning the polar-originating ionospheric current systems. One is the shielding effect of region 2 current on DP2-type magnetic variation (Sect. 3.2) and the other is the examination of the north-south asymmetry of the magnetic variations at the summer solstice (Sect. 3.3).

\section{Equatorial conductivity model}

\subsection{Basic conditions}

The simulation scheme used extensively by many authors so far is the model that assumes that the ionosphere is a conducting thin sheet. In this model, the ionospheric conductivity is obtained by setting the vertical electric current to zero at every point in the ionosphere. Then a set of the equivalent, two-dimensional height-integrated conductivity is derived through algebraic calculations. This model, based on a thin shell approximation of the ionosphere, is usually called 'thin shell model' (the 'thin shell' dynamo model of Forbes and Lindzen, 1976a in essence). The equation of Ohm's law for the ionospheric current in the thin shell model is:

$J=\boldsymbol{\Sigma} \cdot E=-\left(\begin{array}{cc}\Sigma_{\theta \theta} & \Sigma_{\theta \varphi} \\ \Sigma_{\varphi \theta} & \Sigma_{\varphi \varphi}\end{array}\right) \cdot \nabla \Psi$,

where $J$ is the ionospheric height-integrated current density, $\boldsymbol{\Sigma}$ height-integrated conductivity tensor, $E$ electric field, $\theta$ and $\varphi$ colatitude and longitude, and $\Psi$ electric potential, respectively. Each component of $\boldsymbol{\Sigma}$ is the height-integrated values of $\sigma_{\theta \theta}, \sigma_{\theta \varphi}, \sigma_{\varphi \theta}$ and $\sigma_{\varphi \varphi}$, which are determined under the assumption that the vertical current is zero in the ionosphere. The forms of $\sigma_{\theta \theta}, \sigma_{\theta \varphi}$ and $\sigma_{\varphi \varphi}$ are

$\sigma_{\theta \theta}=\frac{\sigma_{0} \sigma_{1}}{\sigma_{0} \sin ^{2} I+\sigma_{1} \cos ^{2} I}$,
$\sigma_{\theta \varphi}=\frac{\sigma_{0} \sigma_{2} \sin I}{\sigma_{0} \sin ^{2} I+\sigma_{1} \cos ^{2} I}$,

and

$\sigma_{\varphi \varphi}=\sigma_{1}+\frac{\sigma_{2}^{2} \cos ^{2} I}{\sigma_{0} \sin ^{2} I+\sigma_{1} \cos ^{2} I}$,

where, $\sigma_{0}, \sigma_{1}$ and $\sigma_{2}$ are longitudinal, Pedersen and Hall conductivities and $I$ dip angle, respectively. $\sigma_{\varphi \theta}$ is exactly the minus of $\sigma_{\theta \varphi}$.

The ionospheric currents caused by the input of fieldaligned currents in the polar region can be obtained by solving the equation of current divergence,

$\nabla \cdot J=\sin I \cdot j_{\|}$,

where, $j_{\|}$is the density of the field-aligned current (positive for downward) and $I$ is the dip angle. The combination of Eqs. (1) and (5) yields a partial differential equation of elliptic type for electric potential, $\Psi$. For the precise equation form, refer to Kamide and Matsushita (1979a). The equation is converted to the difference equation and can be solved numerically by an iteration method.

The distribution of the conductivity should be set as realistically as possible. In this work, the base of the ionospheric conductivity $\sigma_{0}, \sigma_{1}$ and $\sigma_{2}$ are derived in the noon and midnight meridian using IRI-90 and CIRA-72 models. Collision frequencies are deduced on the basis of the tables of Banks and Kockarts (1973), then $\sigma_{\theta \theta}, \sigma_{\theta \varphi}$ and $\sigma_{\varphi \varphi}$ are integrated with height from 80 to $400 \mathrm{~km}$.

The enhancement of the conductivity in the auroral region is added using the model presented by Reiff (1984). As the base model for a geomagnetically quiet period, the conductivity in the auroral region is calculated by using zero to the AE parameter in the model of Reiff (1984). The derived Pedersen conductivity is superposed on $\Sigma_{\theta \theta}$ and $\Sigma_{\varphi \varphi}$ of the IRI model and Hall conductance on $\Sigma_{\theta \varphi} . \Sigma_{\varphi \theta}$ is given as $-\Sigma_{\theta \varphi}$ at this stage. 
For $\Sigma_{\theta \theta}$ which becomes quite large at the equator, the value at the equator is reduced such that the gradient of $\Sigma_{\theta \theta}$ at $1^{\circ}$ latitude becomes equal to that at $2^{\circ}$ latitude. This procedure is similar to that of Tsunomura and Araki (1984) and is not thought to be far from the actual situation because the magnetic field lines may not be perfectly aligned horizontally for the whole height range of the ionosphere in the actual situations.

Second, the modification of $\Sigma_{\varphi \varphi}$ is made. Untiedt (1967) and Richmond (1973) made calculations of the meridional current system in the equatorial region driven by an external eastward electric field. They showed that the strength and width of the heightintegrated eastward ionospheric current are increased compared to those of the $\sigma_{\varphi \varphi}$ model (equivalent to the thin shell model) of Sugiura and Cain (1966). Being based on the same conductivity profiles in height and the same eastward electric field strength, this result leads to the idea that the height-integrated conductivity is enhanced equivalently by the presence of the meridional current system. Both of the meridional current system models of Untiedt (1967) and Richmond (1973) yielded more realistic solutions than those of the $\sigma_{\varphi \varphi}$ model. The existence of the meridional current system was evidenced by the observations by rocket measurements (Musmann and Seiler, 1978) and MAGSAT (Maeda et al., 1982). By making a three-dimensional calculation in the equatorial region, Forbes and Lindzen (1976a, b) showed that the equatorial electrojet strength is nearly doubled and the jet region is widened. Forbes and Lindzen (1976b) gave an explanation to enhance $\Sigma_{\varphi \varphi}$ by the effect of vertical current flow. Hence, it is strongly suggested that the strength and the width of $\Sigma_{\varphi \varphi}$ should be enhanced and broadened, respectively more than those in the thin shell model.

The comparison between the meridional current system model and the thin shell model for the heightintegrated eastward current is shown in Fig. 7 of Untiedt (1967). The result is used to decide the multiplication factor applied for $\Sigma_{\varphi \varphi}$ of the thin shell model. According to Untiedt's (1967) result, $\Sigma_{\varphi \varphi}$ in the equatorial region is multiplied as follows; 1.2 times at the equator, 1.8 times at $1^{\circ}$ latitude, and twice at $2^{\circ}$ latitude. For latitudes higher than $2^{\circ}$, the multiplication factor is linearly decreased with increasing latitude to unity at $25^{\circ}$ latitude.

Figure 1 shows the two-dimensional height-integrated conductivity in the noon-midnight meridian obtained after these procedures for $\Sigma_{\theta \theta}$ and $\Sigma_{\varphi \varphi}$ to the base conductivity setting equinox, 50 for the sunspot number and $0^{\circ}$ for the geographic longitude for the IRI-90 parameters. The pattern is almost same as the conductivity model of Tsunomura and Araki (1984) except for the slight enhancement in the auroral region. The enhancement in the region becomes larger when $\mathrm{AE}$ parameter is increased.

\subsection{Estimate of $\Sigma_{\theta \varphi}$ in the equatorial region}

The treatment of $\Sigma_{\theta \varphi}$ is more complicated and important when discussing the structure of the electric field at the

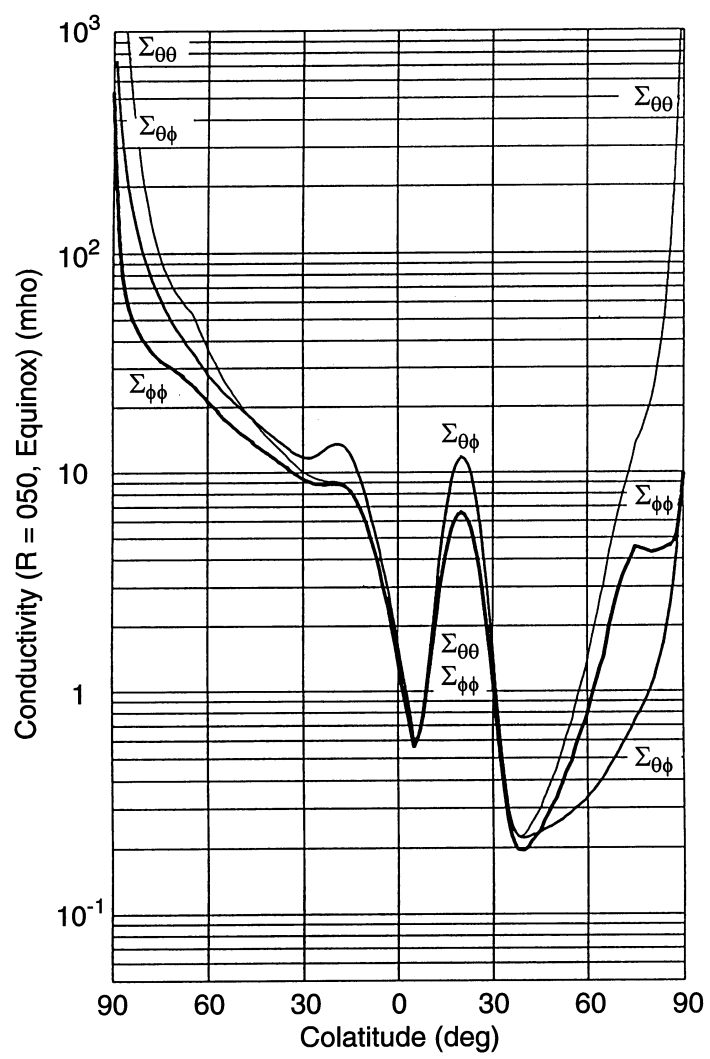

Fig. 1. Noon-midnight profile of $\Sigma_{\theta \theta}, \Sigma_{\theta \varphi}$ and $\Sigma_{\varphi \varphi}$ of the ionospheric height-integrated conductivity tensor for the condition on equinox with sunspot number 50 for IRI parameters. The abscissa is colatitude numbered from -90 at the dayside equator (left side) to 90 at the nightside equator (right side). The large values of $\Sigma_{\theta \theta}$ and zeros of $\Sigma_{\theta \varphi}$ in the equatorial region are not plotted in the figure

equator. In the thin shell model, $\Sigma_{\theta \varphi}$ reaches its maximum very close to the equator and is quite sharply reduced to zero at the equator. It keeps its antisymmetry with respect to the equator. Tsunomura and Araki (1984) made their calculations by setting $\Sigma_{\theta \varphi}$ at $1^{\circ}$ latitude equal to that at $2^{\circ}$ latitude and ignoring its gradient at the equator. Though Tsunomura and Araki's (1984) procedures gave a roughly realistic solution, the modeling of the $\Sigma_{\theta \varphi}$ near the equator should be discussed to make the physical base more definite. In addition their formulation cannot be applied to the calculation covering both hemispheres simultaneously.

Before the discussion of $\Sigma_{\theta \varphi}$, the original equations to derive Eq. (3) should be reexamined. The equation for $j_{\theta}$ is written as;

$$
\begin{aligned}
j_{\theta}= & \left(\sigma_{0} \cos ^{2} I+\sigma_{1} \sin ^{2} I\right) \cdot E_{\theta}+\sigma_{2} \sin I \cdot E_{\phi} \\
& +\left(\sigma_{0}-\sigma_{1}\right) \sin I \cos I \cdot E_{z},
\end{aligned}
$$

where, $z$ denotes the vertical upward direction to form the three-dimensional, orthogonal coordinate system $\theta \varphi z . E_{z}$ is written as:

$E_{z}=\frac{-\left(\sigma_{0}-\sigma_{1}\right) \sin I \cos I \cdot E_{\theta}+\sigma_{2} \cos I \cdot E_{\phi}}{\sigma_{0} \sin ^{2} I+\sigma_{1} \cos ^{2} I}$. 
The contributions of the original east-west component of electric field to the north-south component of electric current as shown in the second term of the right side of Eq. (6) appears explicitly as long as $E_{\varphi}$ exists. Here, the contribution through the vertical field, $E_{z}$ as described in the second term of the numerator of the right side of Eq. (7), and then the third term of the right side of the Eq. (6) should be examined.

In the thin shell model, the algebraic calculation is operated assuming that the vertical current is zero. This procedure yields a vertical, polarization electric field as an algebraic product. The vertical component includes the parallel component for the magnetic field line of force off the equator and then results in the north-south component of the ionospheric current through the longitudinal conductivity, $\sigma_{0}$. This is the mechanism for producing large values of $\sigma_{\theta \varphi}$ near the equator.

The problem is that the parallel component of electric field given by this automatic algebraic calculation should be much larger than that of the real nature. In reality the parallel component of the electric field should be kept almost at zero because of the high conductance in the direction of the magnetic field line. Note that this discussion is applied only for the polarization field, $E_{z}$. It is not necessary to reevaluate the external north-south component of electric field that contributes directly, such as the first term of the right side of Eq. (6). The expectation that the polarization electric field in the north-south direction is weak is supported by the numerical analysis of the meridional current system by Richmond (1973). The potential contours in the meridional plane, which are produced by giving the eastward electric field as the driving source, were almost parallel to the magnetic lines of force in Richmond's (1973) result. The thin shell model does not consider this situation and then leads to a large value of $\Sigma_{\theta \varphi}$ just near the equator.

Therefore, the contribution of the parallel part of the $E_{z}$ as described in the second term of the numerator of the right side of Eq. (7) should be reduced to give the more realistic values of $\Sigma_{\theta \varphi}$ near the equator. This process can be understood as another effect of the meridional current system.

As the reduction factor cannot be decided theoretically, a trial and error method is performed to find the solution. The contribution of the second term of the numerator of Eq. (7) is estimated by making calculations using the conductivity models changing the reduction of the term. The models reducing the effect of the term from Eq. (3) in the course of height integration by the rate of $0.0,0.5,0.75$ and 1.0 are derived for comparison. In each model the reduction rate is maximum just at the neighborhood of the equator and decrease linearly with increasing latitude to zero at $30^{\circ}$ latitude. The noon-midnight profiles of $\Sigma_{\theta \varphi}$ with the maximum reduction rate, $0.0,0.5,0.75$ and 1.0 are shown in Fig. 2. The model 0.0 corresponds to the original profile. Note that $\Sigma_{\theta \varphi}$ shows the maximum just next to the equator $\left(1^{\circ}\right.$ latitude in the figure) except for the full reduction case (the reduction rate is 1.0). The latitudinal gradient of $\Sigma_{\theta \varphi}$ at $1^{\circ}$

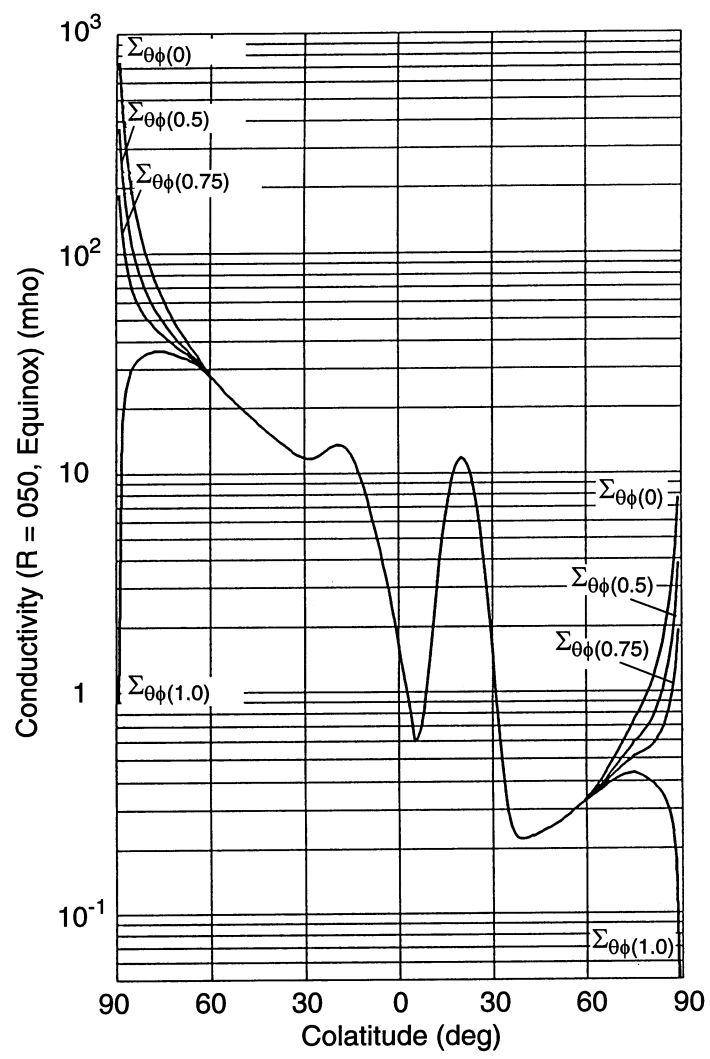

Fig. 2. Noon-midnight profiles of $\Sigma_{\theta \varphi}$ with maximum reduction rates of $0 \%\left(\Sigma_{\theta \varphi(0.0)}\right), 50 \%\left(\Sigma_{\theta \varphi(0.5)}\right), 75 \%\left(\Sigma_{\theta \varphi(0.75)}\right)$ and $100 \%\left(\Sigma_{\theta \varphi(1.0)}\right)$ for the $E_{z}$ contribution in the longitudinal direction. The IRI parameters and the plot style are same as those for Fig. 1

latitude is zero for the models of the $0.0,0.5$ and 0.75 reduction rates. Assuming the viewpoint that the parallel component of electric field along the magnetic lines of force should be small, the full reduction case is expected to be most realistic. It is worth noting that the pattern of $\Sigma_{\theta \varphi}$ of the full reduction case is similar to that of Fejer (1953) except for the difference in the peak latitude.

This discussion was devoted to the feature of polarization electric field $\left(E_{z}\right)$ driven by the external east-west component of the electric field $\left(E_{\varphi}\right)$. Only the conductivity components associated with $E_{\varphi}$, that is, $\Sigma_{\theta \varphi}$ and $\Sigma_{\varphi \varphi}$, are modified. The reduction of $\Sigma_{\theta \varphi}$ and the enhancement and broadening of $\Sigma_{\varphi \varphi}$ from the thin shell model are the products obtained by allowing the vertical current due to the meridional current system driven by $E_{\varphi}$.

It is difficult to decide whether a meridional current system is driven by an external north-south component of the electric field $\left(E_{\theta}\right)$. Untiedt (1967) briefly discussed this matter and showed that $E_{\theta}$ does not drive a meridional current so much. This means that the modulation of $\Sigma_{\varphi \theta}$ from the thin shell model is not necessary. Therefore, the procedure used for $\Sigma_{\theta \varphi}$ is not used for $\Sigma_{\varphi \theta}$. In reality, the calculated results of the ionospheric electric field and current were not so much changed by using the similar modulation for $\Sigma_{\varphi \theta}$ because the relating component of the electric field $\left(E_{\theta}\right)$ is primarily very small. 


\subsection{Comparison of different models of $\Sigma_{\theta \varphi}$}

For the four conductivity models, the electric field and current are calculated giving a current source in high latitudes. The distribution of the field-aligned current is given similarly as that of Kamide and Matsushita (1979a) as follows;

$j_{||}= \pm j_{0} \exp \left[-\frac{\left(\theta-\theta_{0}\right)^{2}}{D_{\theta}^{2}}-\frac{\left(\varphi \mp \varphi_{0}\right)^{2}}{D_{\varphi}^{2}}\right]$,

where, the upper and lower signs of $j_{0}$ and $\varphi_{0}$ are taken for the currents flowing into or away from the ionosphere. The parameters, $\theta_{0}, \varphi_{0}, D_{\theta}, D_{\varphi}$ and $j_{0}$ are $15^{\circ}$, $105^{\circ}, 2^{\circ}, 59^{\circ}$, and $10^{-6} \mathrm{~A} / \mathrm{m}^{2}$, respectively.

The equation was solved by the SOR method with a grid spacing of $1^{\circ}$ for the direction of $\theta$ and $7.5^{\circ}$ for $\varphi$. Since the conductivity changes very rapidly near the equator, the grid spacing in $\theta$ direction is set at $0.5^{\circ}$ in the region from the equator to $10^{\circ}$ latitude. The narrowing of the grid spacing in this region is primarily important to obtain the true result. A drastic change of the numerical solution was seen by narrowing the grid spacing from $2^{\circ}$ to $1^{\circ}$ in the equatorial region; the sense of the electric field at the equator was almost reversed. The solution is not changed so much by the change of the grid spacing from $1^{\circ}$ to $0.5^{\circ}$. The electric field strength at the equator is slightly enhanced for the latter. The solution becomes constant when spacing is narrower than $0.5^{\circ}$. I checked it by comparing the results of $0.5^{\circ}$ with those of $0.25^{\circ}$ and $0.1^{\circ}$ settings. In this work, $0.5^{\circ}$ spacing was adopted.

The narrowing of the grid spacing in other regions is not so important as in the equatorial region. The result obtained by setting the grid spacing $0.5^{\circ}$ for whole area is almost the same with that obtained limiting the area in the equatorial region. Note that the latitude at which $\Sigma_{\theta \varphi}$ and $\Sigma_{\varphi \theta}$ take their maxima is $0.5^{\circ}$, that is, very close to the equator except for $\Sigma_{\theta \varphi}$ in the full reduction case.

The boundary condition for the electric potential at the equator is $\mathrm{d} \Psi / \mathrm{d} \theta=0$, and at the pole $\Psi=$ (the mean of $\Psi$ at $89^{\circ}$ latitude circle). The diurnal variation form of conductivity is assumed to be a sine curve from 04-20 LT; the form is basically equivalent to that of Tarpley (1970). The difference between local time (LT) and magnetic local time (MLT) and/or the difference between the geographic and the geomagnetic poles are not considered. The convergence of the calculation is decided by monitoring the flatness of the iterated values of the electric field and current at several points. The number of iterations to obtain the results in this section is 150000 .

Local time profiles of electric and magnetic fields at $60^{\circ}, 30^{\circ}$ latitudes and the equator for the four models are compared in Fig. 3a,b. For the north-south components near the equator, the data at $5^{\circ}$ latitude are shown because they almost disappear close to the equator. The magnetic fields are for the equivalent currents, which are obtained by adding the magnetic fields due to the field-aligned currents to those of the overhead, ionospheric currents. The magnetic fields due to the field-aligned currents are calculated numerically assuming the geomagnetic dipole configuration of the magnetic lines of force. Near the equator, the ionospheric contributions are calculated using Biot-Savart's law for the ionospheric currents between $5^{\circ}$ north and south, because the electrojet current flows in the narrow region and the approximation of the overhead current sheet is broken. The ground induction effect is neglected.

It is clearly seen that electric and magnetic fields at $60^{\circ}$ latitude are not affected by the $\Sigma_{\theta \varphi}$ modulation in the equatorial region. The variation pattern of the magnetic field in $60^{\circ}$ latitude is almost identical with the local time distribution of the variation sense of SCs as shown by Matsushita (1962). Although the full reduction case shows brief difference from other models at $30^{\circ}$ latitude in the dayside, the profiles at $60^{\circ}$ and $30^{\circ}$ latitudes are, in general, similar to the results of Tsunomura and Araki (1984). I would like to add that the profiles of the electric and magnetic fields in low latitudes are much more changed if there is no equatorial enhancement. That is confirmed by the test calculation by making all conductivity equal from $30^{\circ}$ latitude to the equator. The profile of the east-west component of electric field $\left(E_{\varphi}\right)$ at $30^{\circ}$ is consistent with the local time profile of the polarity of MFD of SCF in the observations of HF Doppler frequencies associated SC as shown by Kikuchi et al. (1985).

At the equator, the $E_{\varphi}$ varies considerably by modification of $\Sigma_{\theta \varphi}$, resulting in the drastic change of magnetic fields. It can be seen that the southward component of the electric field $\left(E_{\theta}\right)$ in the morning gradually decreases as the reduction rate increases, whereas the $E_{\varphi}$ increases. The equatorial electric fields in the dayside for cases apart from the full reduction are small and negative. The curves of the electric field for the cases of $0.0,0.5$ and 0.75 cross the zero line twice in the afternoon; it means that a singular point appears in the afternoon for the potential at the equator in these models. This feature of $E_{\varphi}$ at the equator is attributed to the fact that $\Sigma_{\theta \varphi}$ produces a sharp maximum very close to the equator.

Latitudinal profiles of the $\mathrm{H}$ component of magnetic field at $12 \mathrm{LT}$ are shown in Fig. 4. The curves, except for the full reduction case, do not show the equatorial enhancement pattern. The profile of the full reduction case is in good agreement with the mean latitudinal profile of the SC amplitude shown by Rastogi (1993) and that of SC* shown by Rastogi and Sastri (1974). The latitudinal profile from high latitudes to the equatorial region for the full reduction case shows a similar profile to that of Pi2 shown by Yumoto et al. (1994) and Yumoto et al. (1995).

The results, except for the full reduction case, are far from the observed result. It is thought that the full reduction case is the nearest to the realistic condition among the four models. In the next section, comparisons with other models or observational results for SC will be made first for the full reduction case and then some applications using the present model will be presented. 

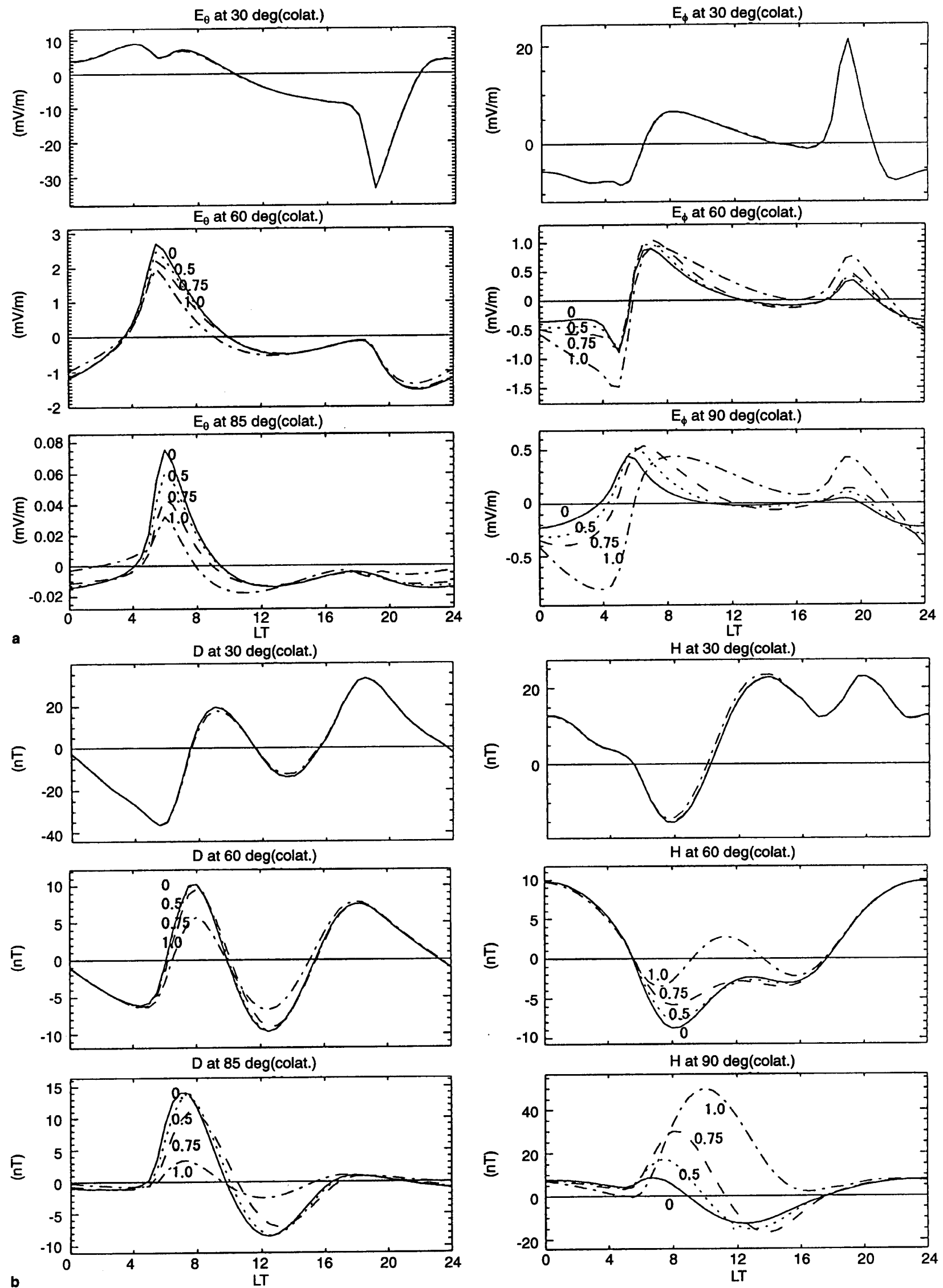
Fig. 3. a Local time variations of southward $\left(\mathrm{E}_{\theta}\right.$, left panels $)$ and eastward $\left(E_{\varphi}\right.$, right panels $)$ components of the ionospheric electric fields at $60^{\circ}, 30^{\circ}$ latitudes and the equator calculated for the four conductivity models. Solid, dotted, dashed and dot-dashed lines are the results with $\Sigma_{\theta \varphi}$ of $0.0,0.5,0.75$ and 1.0 reduction, respectively; numbers labeled on the curves mean the reduction rate. Note that four curves are almost the same in the uppermost panel. b Same as a for D (left panels) and $\mathrm{H}$ (right panels) components of the magnetic fields

\section{Results and discussion}

\subsection{Electric and magnetic fields of the full reduction case}

In Fig. 5, local time profiles of the equatorial electric field and current for the full reduction case and those of Tsunomura and Araki (1984) and 'the initial phase' of Senior and Blanc (1984) are shown; they are obtained for similar sources in high latitudes. The results are arranged so as to equalize the signs and total amounts of the input source current or potential drops in the polar region. Since Senior and Blanc (1984) treated the equator as a conducting belt with $10^{\circ}$ latitude width, the electric current shown here is the average of their result. As the main purpose of Senior and Blanc's (1984) study was the time development of the magnetosphereionosphere coupling process, they did not discuss the latitudinal profile of electric current in low latitude. Therefore, the exact comparison of the current magnitude or latitudinal profile with their result is impossible.

It is seen that the present result and that of Senior and Blanc (1984) take the maximum and minimum at similar local times. Sastri et al. (1993) showed that the magnitude of SC-associated electric field becomes highest near 03 LT. The local time of the morning minimum in the models of Senior and Blanc (1984) and the present one is nearer than that of Tsunomura and Araki (1984)

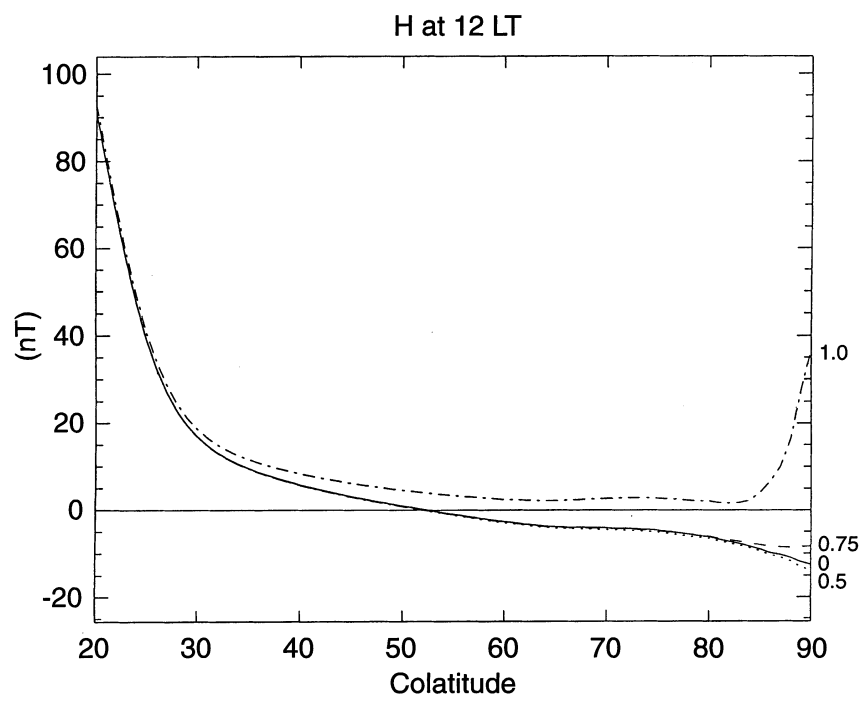

Fig. 4. Latitudinal variations of $\mathrm{H}$ component of magnetic fields in the 12 LT meridian calculated for the four models of $\Sigma_{\theta \varphi}$. Meanings of the labels are same as Fig. 3
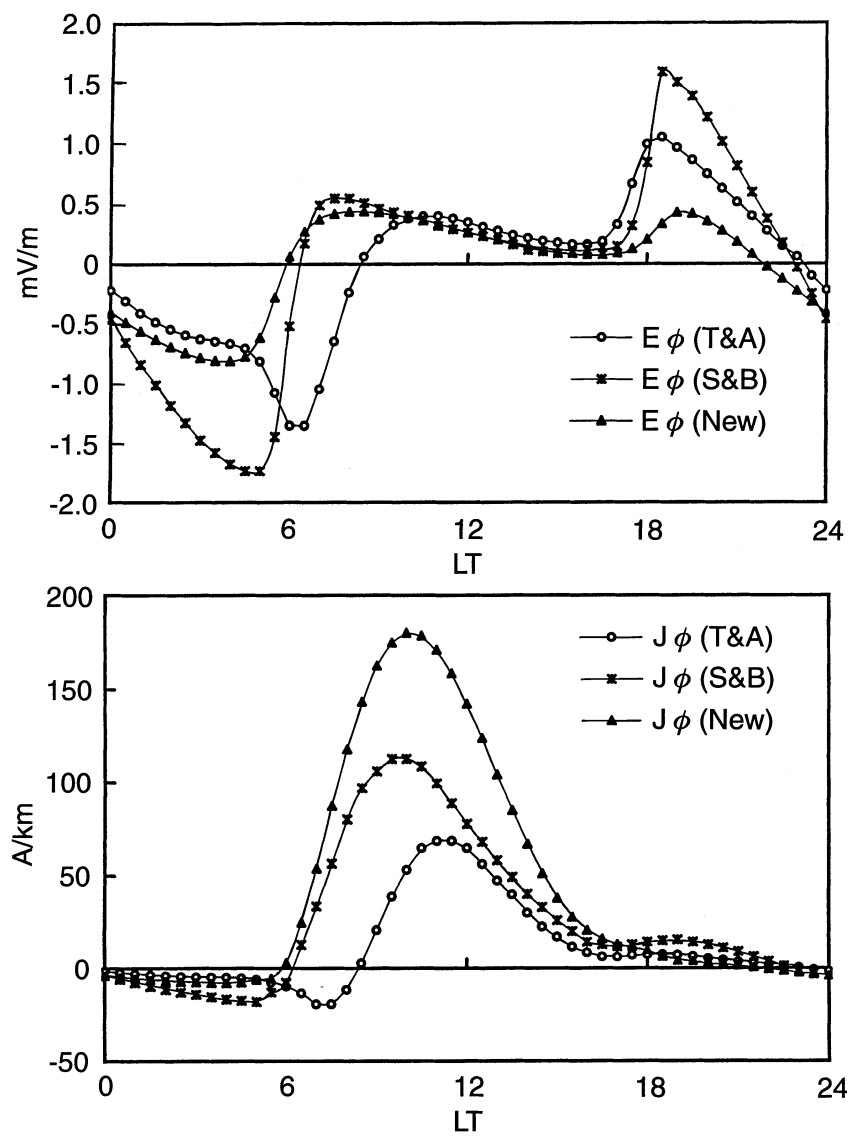

Fig. 5. Comparison of the local time variations of eastward electric field and current at the equator of the present model ('New') with those of Senior and Blanc (1984) (' $S \& B$ ') and Tsunomura and Araki (1984) (' $T \& A$ ')

to the observational result. The local time profile of the electric field in the present model is also in good agreement with those of the prompt penetration zonal electric fields obtained by the radar observation at Jicamarca and the prediction by the Rice convection model shown by Fejer and Scherliess (1997). Thus, the present model clearly shows some improvement in deriving the equatorial electric fields from that of Tsunomura and Araki (1984).

The peak electric field intensity in the morning and evening is smaller in the present model than Senior and Blanc's (1984) result. It cannot be decided which model fits the observations better. It is noted that the similar electric field pattern with sharp maximum in the evening as those of Tsunomura and Araki (1984) and Senior and Blanc (1984) is also derived using the present model setting the grid spacing at $1^{\circ}$ in the equatorial region. However, as noted in the last section, the grid spacing of $1^{\circ}$ is not sufficient to get the true solution.

The electric field intensities in the daytime are nearly the same for all the models. Since the ionospheric conductivity is the highest in the present model, the electric current intensity in the dayside is strongest for the present model than others. The validity of the current intensity of the present model should be clarified in future by the global magnetic and radar observations. At 
the actual comparison with observations, the geomagnetic latitude of the magnetic observation point should be checked carefully because of the rapid latitudinal change of the current intensity near the equator.

Reddy et al. (1981) showed the relationship between the electric and magnetic fields for some SC events. It is difficult to compare the present result with their's exactly because the relationships between the electric and magnetic fields should be examined by extracting the $\mathrm{DP}_{\mathrm{MI}}$ field contribution from the magnetic observations. The local time profile of the mean ratio of the $\mathrm{DP}_{\mathrm{MI}}$ to DL fields, presented by Papamastorakis et al. (1984) can be used for this purpose. Applying Papamastorakis et al.'s (1984) result to the magnetic data of Reddy et al. (1981), the $\mathrm{DP}_{\mathrm{MI}}$ contributions in the events are roughly extracted. Also assuming that the SCassociated $\mathrm{DP}_{\mathrm{MI}}$ electric field is proportional to the deviation of the Doppler frequency, the ratio of the magnetic variation (nT unit) to the electric field variation $(\mathrm{mV} / \mathrm{m}$ unit) for each event of their study can be derived. The ratios calculated for the SC events on December 27, 1979, February 14, 1980 and March 12, 1979, which were analyzed by Reddy et al. (1981), are between $70-130,50-120$ and $30-280$, respectively. The corresponding values derived from the present model are about 110, 80 and 80 , respectively. The values for the present result are near the medians of those inferred from the events analyzed by Reddy et al. (1981).

Sastri et al. (1993) evaluated the equatorial electric fields in the pre-midnight hours associated with two SC events. In this local time range, the SC-associated electric field is expected to vary very rapidly with local time, as shown by Figs. $3 \mathrm{a}$ or 5 . It is also very difficult to compare their results with the present one because the derivation of the pure $\mathrm{DP}_{\mathrm{MI}}$ field from the magnetic observation is very difficult for the events analyzed by them. Roughly comparing the present result with the SC on January 01, 1992, the intensity of the electric field in the present result seems to be same as or a little smaller than their observational result.

The intense electric field was observed near the equator in the premidnight hours associated with a sudden expansion event (Sastri et al., 1995). Although the comparison of this event with the present result is also quite difficult, the observed value is roughly estimated to be several times as large as that of the present result. However, the local time variation of the electric field is very sharp in this local time range. If the input source current pattern in the polar region is somewhat shifted to the morning side, the more intense electric field can be expected from the present calculation as can be seen in Fig. 3a or 5 .

The local time variation of the equatorial enhancement pattern of magnetic field for SC shown by Sarma and Sastry (1995) is different from the present result. Their result shows the peak at $12 \mathrm{LT}$, being different from $10 \mathrm{LT}$ in the present result. However, a similar investigation by Papamastorakis et al. (1984) shows an almost identical result with the present one. Since Sarma and Sastry (1995) did not subtract the contribution of the DL field, it cannot be decided whether their result is inconsistent with the present one or not. Therefore, it can be said that the present result basically agrees with the observations for the local time profile of the magnetic field.

Viewing these results, it is thought that the full reduction process of $\Sigma_{\theta \varphi}$ primarily gives a realistic twodimensional conductivity distribution in the equatorial region. It is interesting that the local time and latitudinal profiles of the magnetic field for the full reduction case are basically same as those of the three-dimensional model of Takeda (1982). The two-dimensional model can principally provide similar solutions than the threedimensional model as long as the external electric field does not vary with height very much. Although it is desirable that the vertical profile of SC-associated electric field is clarified by observations, it may be very difficult to obtain the height profile of the electric field just at the time of SC. However, the speculation that the external electric field of the distant origin does not vary with height so much in low latitude may not be far from reality.

\subsection{Effect of shielding by region 2 current}

Kikuchi et al. (1996) discussed the equatorial enhancement of the DP2 type magnetic variation as a case study and showed that the equatorial enhancement was recognized at the daytime equator and even region 2 field-aligned current, causing a shielding effect, was expected to exist to some extent. Several authors theoretically estimated the shielding effect of region 2 current for the dawn-to-dusk electric field in the equatorial region associated with the DP2 magnetic fluctuations (Nopper and Calovillano, 1978; Senior and Blanc, 1984; Denisenko and Zamay, 1992). However, these authors did not discuss the latitudinal profile of the magnetic fields. It is worth examining the degree of the equatorial enhancement in the latitudinal profile, giving the shielding effect of region 2 current.

Calculations changing the ratio of total amount of region 2 current to that of region 1 are performed for this purpose. The distribution of the region 1 current is same as the previous section. That for the region 2 is different from region 1 only in the peak latitude and the sign. The peak latitude is set at $65^{\circ}$ and the sign of region 2 is reversed with respect to that of region 1 .

The latitudinal profiles of the magnetic field at $12 \mathrm{LT}$ for the three cases are compared in Fig. 6. The latitudinal profile for the case of 0.0 ratio is nearly the same as that shown in Fig. 7 of Kikuchi et al. (1996). However, the enhancement rate of the magnetic variation at the equator with respect to that in high latitudes is a little higher in the case of 0.0 ratio than that of Kikuchi et al.'s (1996) result. Therefore, at the event analyzed by Kikuchi et al. (1996), the ratio of the region 2 to 1 currents is thought to be between 0.0 and 0.5 and may be near 0.0 .

As shown already, the equatorial enhancement ratio, that is the ratio of the amplitude of magnetic disturbance field at the equator to that in high latitude, is possibly 


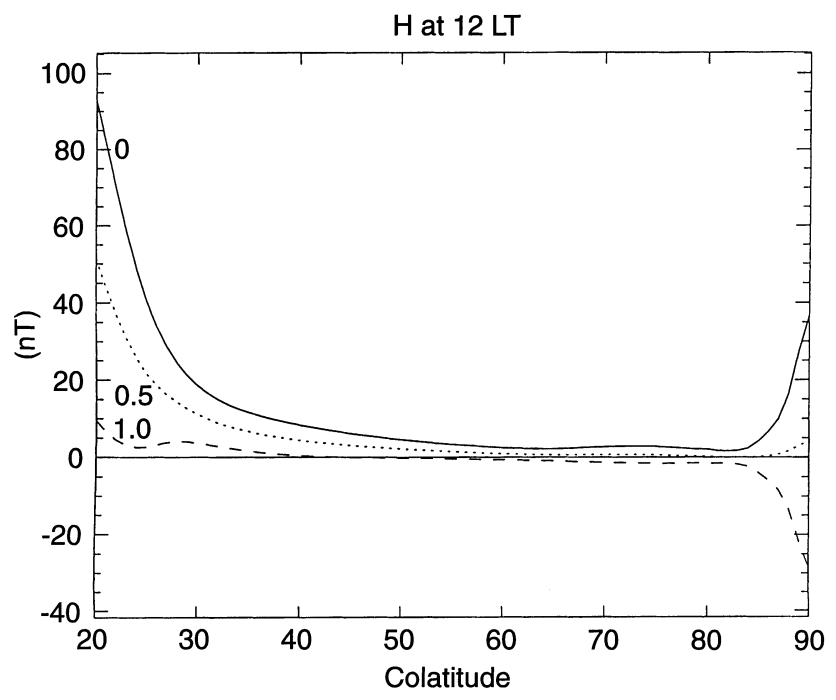

Fig. 6. Latitudinal variations of $\mathrm{H}$ component magnetic fields in the 12 LT meridian calculated for the three models of source current composition. Solid, dotted and dashed lines are for the results with the ratio of region 2 current intensity $0.0,0.5$ and 1.0 to that of region 1 current, respectively
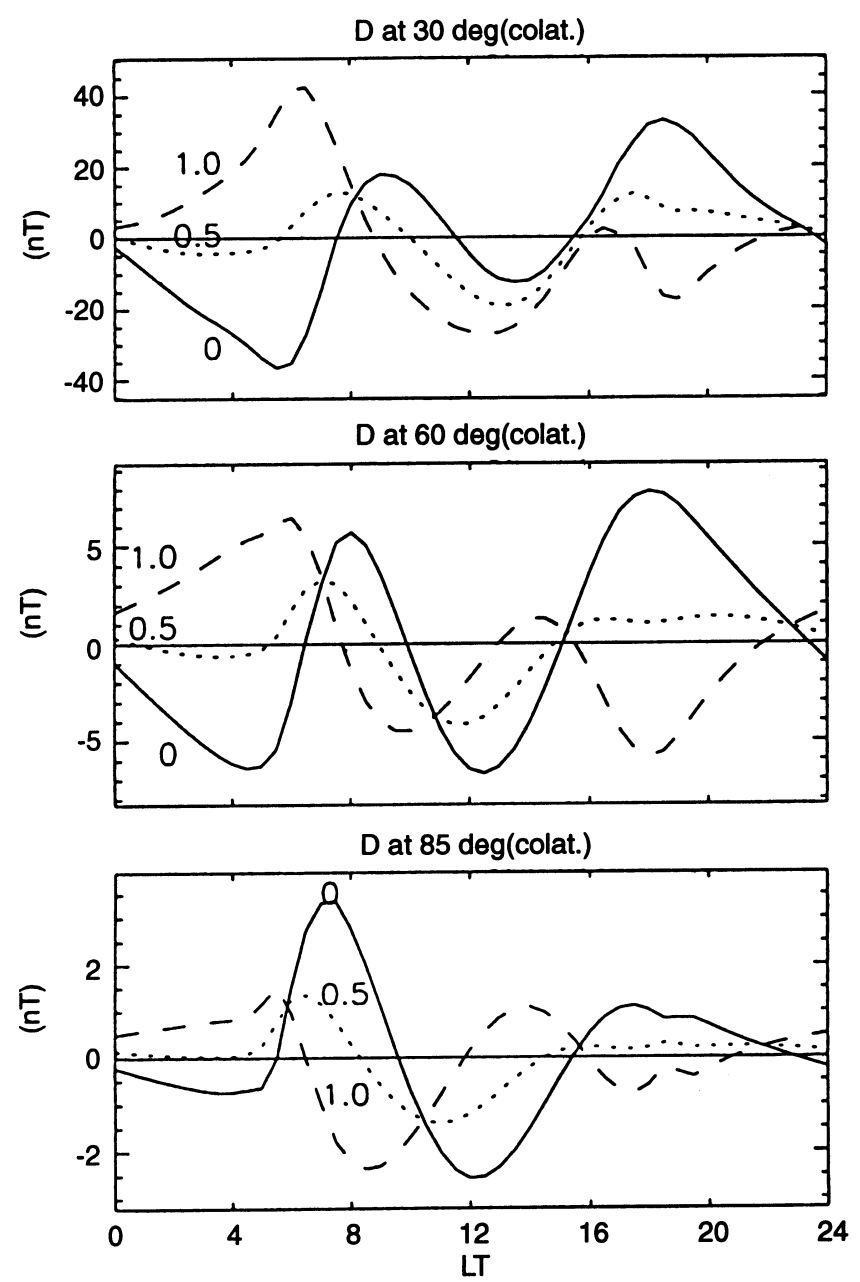

useful to evaluate the mixing ratio of the region 1 and 2 currents. It should be kept in mind, however, that the local time profile of the magnetic variations in high latitudes is very complicated as seen in Fig. 7. Because of the limited number of observation sites, the idealized station pairs cannot be always obtained. The equatorial enhancement rate can be changed very much by event or local time and is not a stable parameter to use as an indicator of the mixing ratio of the region 1 and 2 currents. The polarities of the $\mathrm{H}$ component in middle latitudes in the nightside and/or at the equator in the dayside may be the better indicators for this purpose.

\subsection{Asymmetry of ionospheric current system in solstice}

The relationships of amplitudes, phases and other parameters for geomagnetic phenomena between the Northern and Southern Hemispheres are important to examine the characteristics of the origin of the magnetic variations. For example, if the magnetic variation at an observation site in the summer hemisphere is larger than that at its conjugate point in the winter one, it is expected that the driving force has some relationships with the ionospheric currents. This is because the
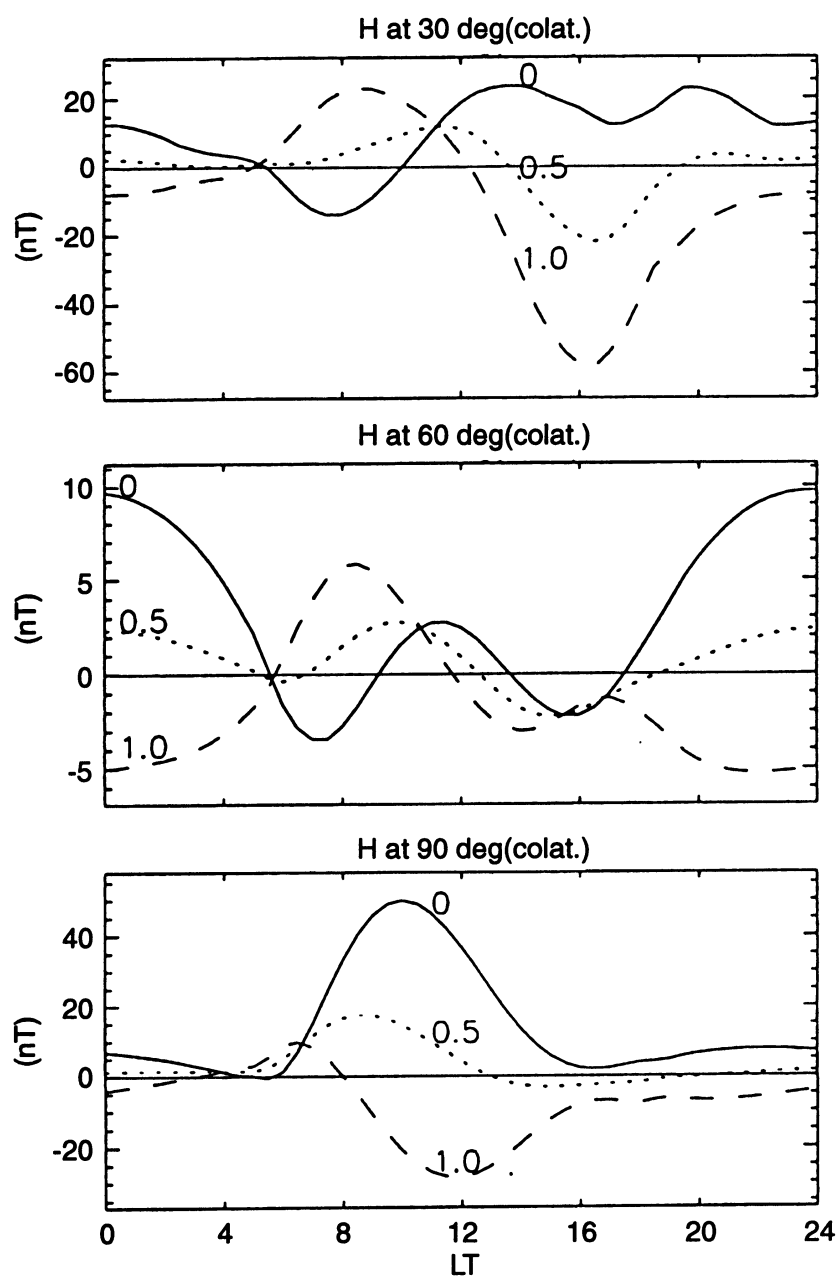

Fig. 7. Local time variations of $\mathrm{D}$ (left panels) and $\mathrm{H}$ (right panels) components of the magnetic fields at $60^{\circ}, 30^{\circ}$ latitudes and the equator calculated for the three models of source current composition. Labels as in Fig. 6 
background ionospheric conductivity due to solar radiation is higher in the former. However, the dependence on the ionospheric conductivity may be different between the voltage generator and the current one, as discussed for the quiet time geomagnetic variations by Fujii et al. (1981) and Fujii and Iijima (1987). The northsouth asymmetry is not basically expected for the current generator, such as discussed by Vickrey et al. (1986) for Birkeland currents of intermediate scale size. It is useful to examine the difference of the asymmetry characteristics for the voltage generator and the current one by a numerical analysis.

Kamide and Matsushita (1979a) and Nisbet et al. (1978) calculated the ionospheric current system driven by a current generator for one hemisphere independently, setting the solstice condition for the ionospheric conductivity. In this study, after making the universal conductivity model, numerical calculations of the ionospheric currents covering both hemispheres simultaneously can be operated regarding the equator as an intermediate point.

Conductivity profiles in the Northern and Southern hemispheres are given as the summer and winter conditions of ionospheric conductivity derived from IRI-90 model setting the sunspot number as 50 (Fig. 8). Conductivity enhancements in the auroral region are same as those in Sect. 2.1 for both hemispheres. Since the conductivity at the equator for the summer and the winter conditions are not so different, the averaged values are used at the equator.
The types of the source are two types, that is, the voltage and the current generators. For the voltage generator, the potentials at $75^{\circ}$ latitude are given a sinusoidal form, the peaks of which are located at 06 and $18 \mathrm{LT}$. The total potential drop is $100 \mathrm{kV}$, giving $+50 \mathrm{kV}$ at $06 \mathrm{LT}$ and $-50 \mathrm{kV}$ at $18 \mathrm{LT}$. The property of the current generator is same as that of Sect. 2.3. As the peaks of the calculated potentials for the current generator are situated near 06 and $18 \mathrm{LT}$, the results can be easily compared.

The calculations do not include any feedback to make the potentials of conjugate points equal. In the actual state, the potential difference between the conjugate points may drive the secondary field-aligned currents to equalize the potentials. However, the process is expected to be complete after the transition time of the signal through the magnetic lines of force. There may be some delay of the order of a few minutes after the impression of the external electric field or current. Since the instantaneous response of the ionosphere to the external driver is discussed in this work, the feedback process is neglected.

Figure 9 shows potential contours of the voltage generator. The potential profiles are almost symmetric with respect to the equator as if both hemispheres are connected electrically. It is noted that the potential contours are skewed at the source latitude as if dragged by an external force.

The potential contours for the current generator are shown in Fig. 10. The total potential drops are $69.3 \mathrm{kV}$
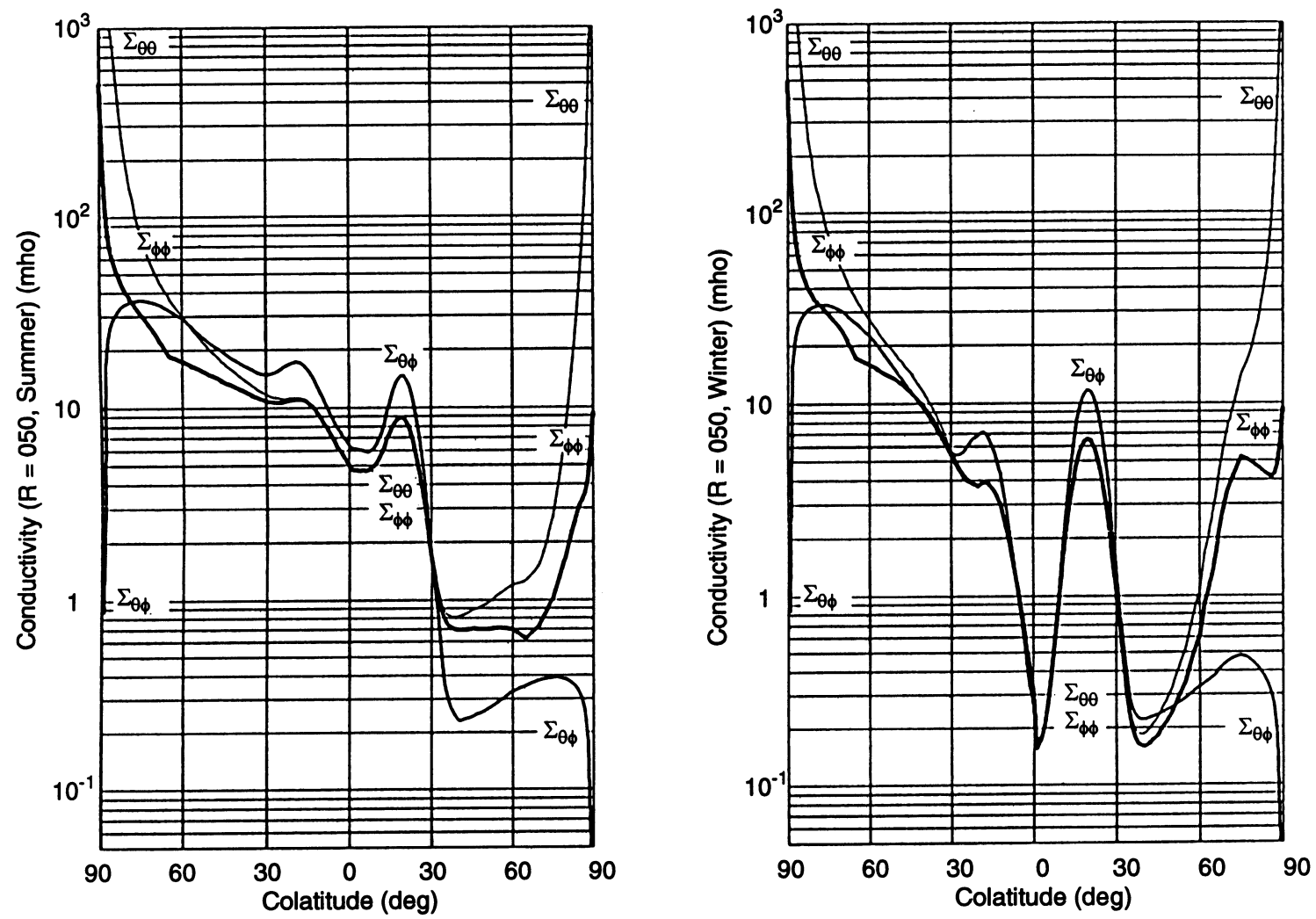

Fig. 8. Noon-midnight profiles of $\Sigma_{\theta \theta}, \Sigma_{\theta \varphi}$ and $\Sigma_{\varphi \varphi}$ of the ionospheric height-integrated conductivity tensor for the conditions of summer (left) and winter (right) solstices with sunspot number 50 for IRI parameters. The meanings of the labels and the plot style are as for Fig. 1 
and $148.4 \mathrm{kV}$ in the summer and the winter hemispheres, respectively. The average of them $(108.85 \mathrm{kV})$ is a little larger than that of the voltage generator. The peak potential values are almost same as that calculated for each hemisphere independently setting the conductivity models in the summer or winter solstice. The peak positions of the electric potential are not shifted to the nightside because the contrast of the day-night conductivity in high latitudes is reduced due to the slight enhancement of the auroral conductivity. It is clear that the difference in the calculation schemes, simultaneous for both hemispheres or independently for each hemisphere, does not make much difference in the electrical conditions in high latitudes.

In Fig. 11, the average latitudinal profiles of the magnetic fields for both the generators are compared for 06-12 and 12-18 LT blocks. Generally the magnetic fields are larger for the voltage generator than the
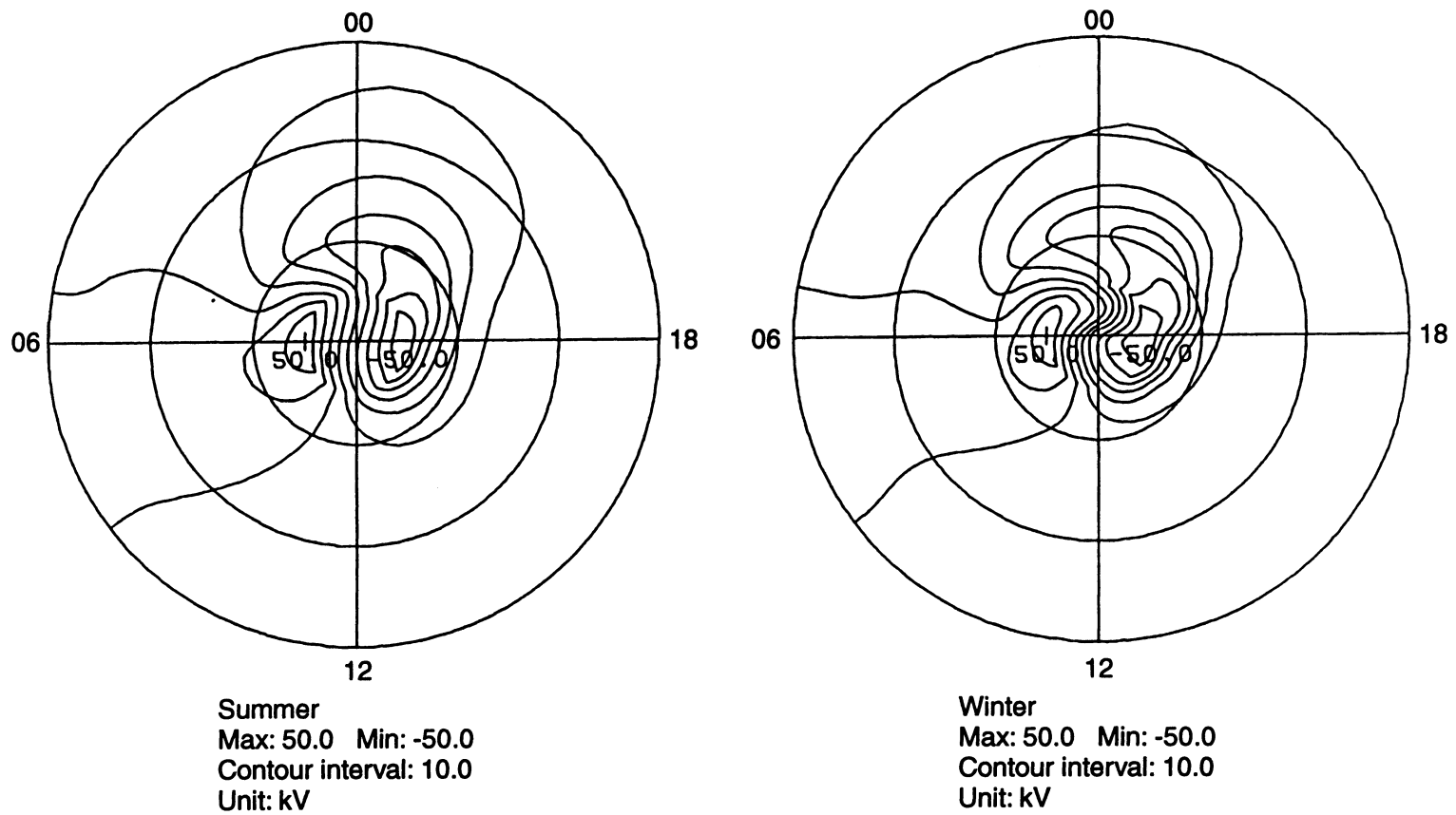

Fig. 9. Potential contours in the summer (left) and winter (right) hemispheres for the voltage generator. Circles are $60^{\circ}$ and $30^{\circ}$ latitudes and the equator from the inside, respectively. The interval of the potential contours is $10 \mathrm{kV}$

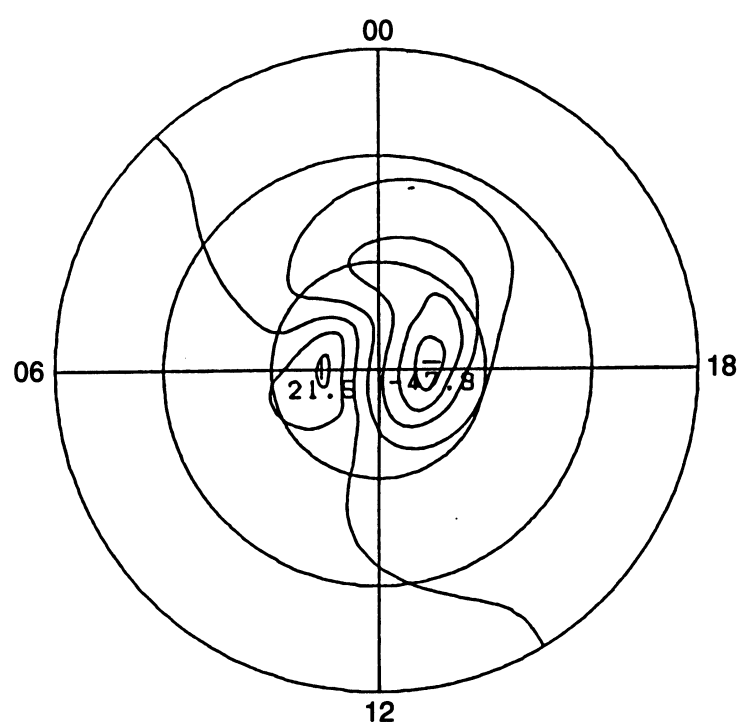

Summer

Max: 21.5 Min: -47.8

Contour interval: 10.0 Unit: kV

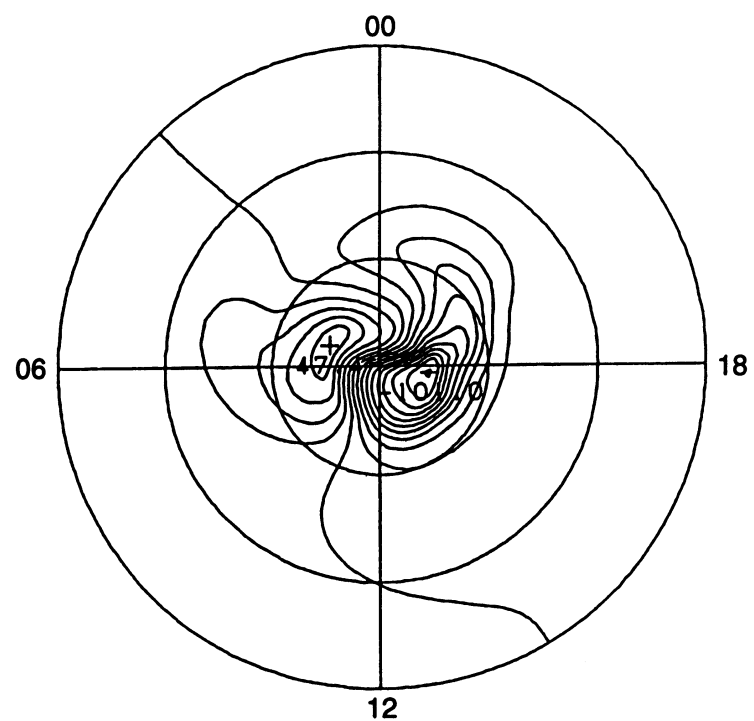

Winter

Max: 47.4 Min: -101.0

Contour interval: 10.0

Unit: kV

Fig. 10. Same as Fig. 9 for the current generator 

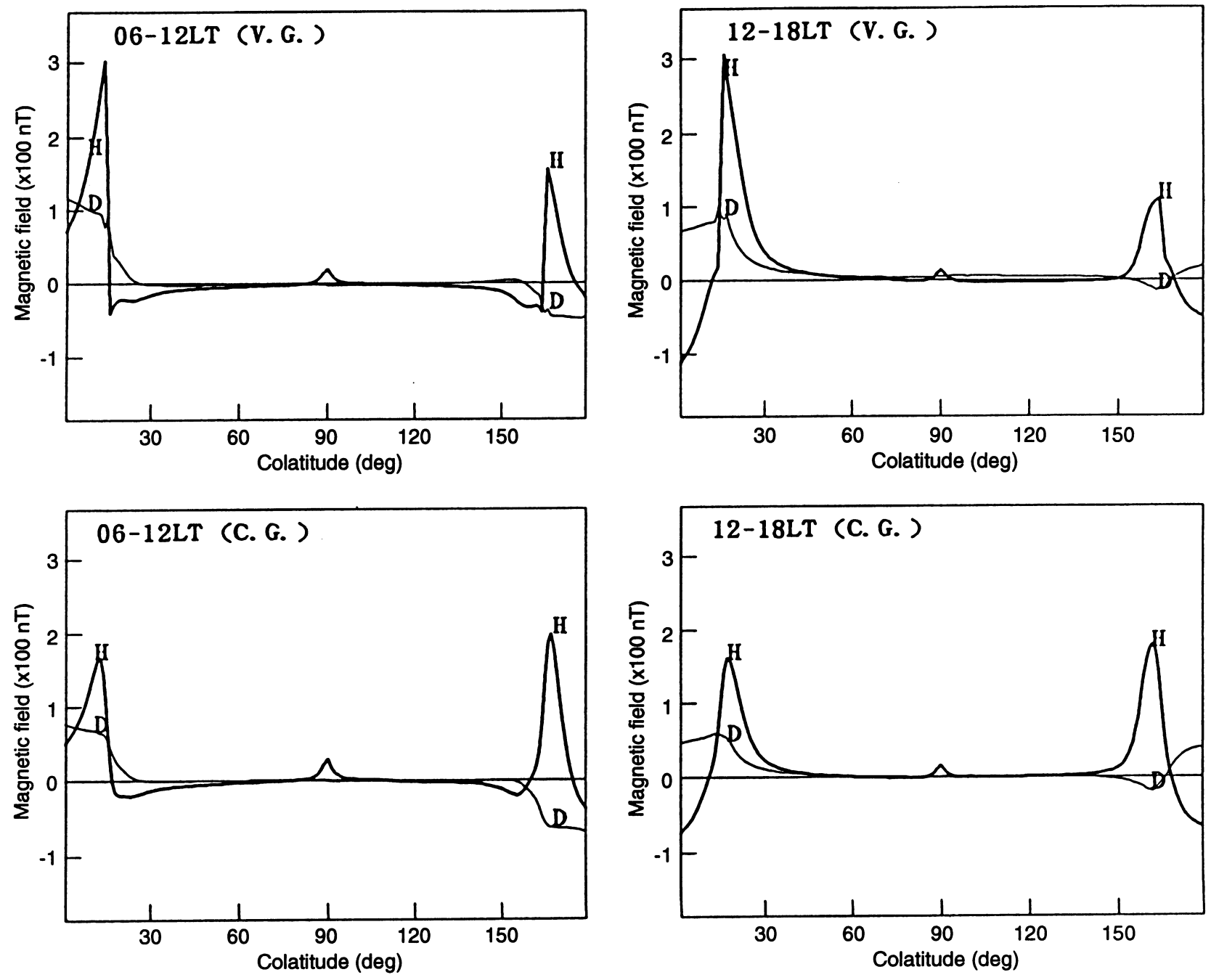

Fig. 11. Average latitudinal variations of $\mathrm{H}$ (thick lines) and $\mathrm{D}$ (thin lines) component magnetic fields of the voltage (upper panels) and current (lower panels) generators, for two local time blocks

current generator in high latitudes. The magnetic fields in the polar region are larger in the summer hemisphere than the winter one for the voltage generator, whereas the contrast is not clear or even in the reversed situation for the current generator.

Figure 12 shows the average latitudinal profiles of the magnetic fields for both the generators in the latitude range from $60^{\circ}$ to $-60^{\circ}$ for $06-12$ and $12-18$ LT blocks. At the equator, the amplitudes are generally larger in the 06-12 LT block than the 12-18 LT one for both cases. The north-south asymmetry of the amplitudes is clearly seen in the $\mathrm{H}$ component of the 06-12 LT block of the voltage generator (upper right). The asymmetry is not seen in the blocks of the current generator (lower panels). For middle to high latitudes differences due to the sources were not apparent. The magnetic fields in the late morning at the equator are larger in the current generator than the voltage one, even taking into account of the difference in the total potential drops. This is because the diurnal variation pattern of the electric potential for the voltage generator in high latitudes is enforced as a sine curve; it results in the unnatural fittings of the potential contours as can be seen in Fig. 9. I would like to mention that the equatorial magnetic field shown here is different from those obtained by the independent calculations for the solstice conditions; that means the calculation covering both hemispheres is desirable to discuss the ionospheric contribution in low latitudes for solstices.

Comparing SC amplitudes at Syowa and Reykjavik, the conjugate stations in the auroral region, Nagata et al. (1966) showed that the amplitude of the H component often becomes higher in the winter hemisphere than the summer. Tsunomura (1989) reexamined the matter using the magnetic data at the same stations and showed that the $\mathrm{D}$ component in the summer hemisphere is a little larger than that in the winter one. These relationships in the auroral region seem to fit the current generator feature shown in Fig. 11. On the other hand, the amplitude relationship of SC between the summer and the winter hemispheres in the $210^{\circ}$ meridian (Yumoto et al., 1995; Yumoto et al., 1996) are roughly consistent with the results of the 12-18 LT block of the voltage generator (Fig. 12). It is difficult to decide the 

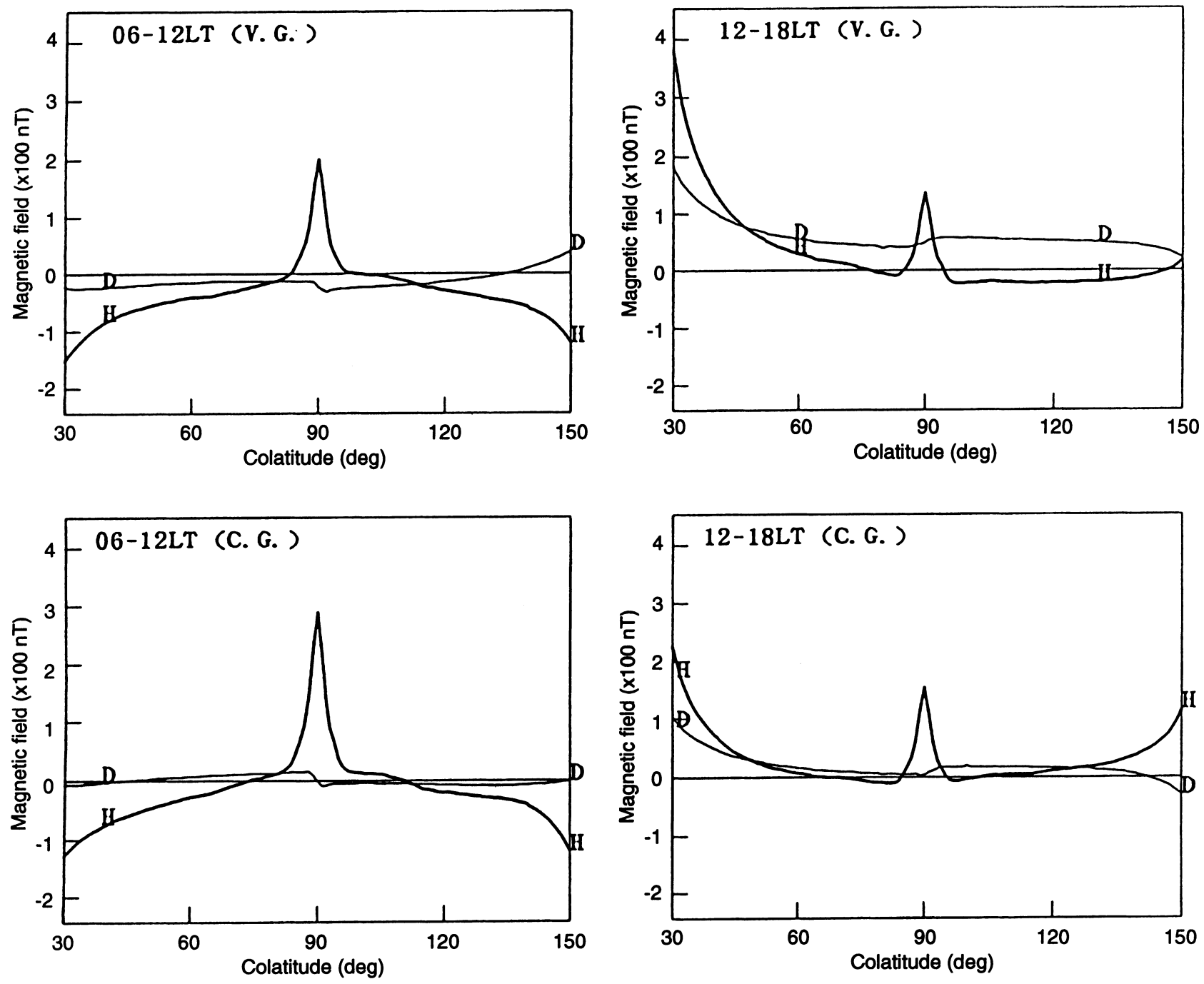

Fig. 12. Same as Fig. 11 for the latitude range from $60^{\circ}$ to $-60^{\circ}$

source type of the $\mathrm{DP}_{\mathrm{MI}}$ of $\mathrm{SC}$, though it is basically expected to be the voltage generator. The actual process may possibly be the intermediate between the voltage and current generators.

Saito et al. (1989) showed the asymmetry of Pc3-5 magnetic pulsations in the auroral region comparing the data at Syowa with those at Husafell. They showed that the powers of Pc3-5 magnetic pulsations are relatively higher at the winter than the summer. They also showed that the amplitude ratio of the pulsations for the stations varies because of the nearly three hour difference in the local time. They suggested the screening effect of the ionosphere as a cause for these relationships. The complete understanding of this matter should be obtained after consideration of other matters, such as the horizontal wavelength or the period of the pulsations. The result obtained here (Fig. 11) may be one of the items to discuss. If the amount of the field-aligned currents associated with the standing oscillation of magnetic field line is thought to be constant, the present result for the current generator may give the base of the background condition.

\section{Conclusion}

I proposed a method to derive a realistic model for the global two-dimensional ionospheric layer conductivity after considering the effect of the meridional current system on the equivalent, ionospheric conductivity. The meridional current system, including the vertical current, contributes the components in the height-integrated conductivity tensor associated with the east-west component of the electric field. It is shown that $\Sigma_{\theta \varphi}$ must be reduced near the equator and $\Sigma_{\varphi \varphi}$ intensified and made to have broad maximum near the equator. The calculated result shows that the modulation of $\Sigma_{\theta \varphi}$ is very effective for the formation of the electric field at the equator. The model with fully reduced $\Sigma_{\theta \varphi}$ reveals the observed feature of SC best.

The full reduction model was applied to two cases:

1. The latitudinal profile of the DP2-type magnetic variation in the daytime is discussed, changing the rate of shielding for the dawn-to-dusk electric field due to the region 2 current. It is shown that the equatorial enhancement would not appear when the ratio of region 
2 to region 1 currents exceeds 0.5 . The result was compared with the observational result of Kikuchi et al. (1996) and the interpretation that the ratio of the region 2 current to the region 1 may be nearly 0.0 is supposed for their case.

2. Differences in the characteristics of the north-south asymmetry of the ionospheric current system between the voltage and current generators are discussed, on the basis of the calculation including both hemispheres simultaneously. It is shown that the magnetic fields in high latitudes show a positive relationship with the conductivity for the voltage generator. However, the relationship is vague or even reversed somewhat for the current generator. The north-south asymmetry of the magnetic fields in middle latitudes is seen only for the afternoon block of the voltage generator.

As shown by these applications, the new model, being based on the International Reference Ionosphere (IRI) model, and less technical treatment to construct the conductivity model, is versatile and may be useful to develop quantitative analysis in some aspects of magnetosphere-ionosphere coupling problems.

Acknowledgements. The author thanks Prof. Araki and Dr. Iyemori of Kyoto University and Dr. Kikuchi of Communications Research Laboratory for encouragement and useful discussions for this study. He also thanks Dr. Takeda of Kyoto University for support to make the ionospheric conductivity model using the computer system of Kyoto University. The calculations were made using Data Processing and Analysis System for Geomagnetism of the Kakioka Magnetic Observatory.

Topical Editor D. Alcaydé thanks M. Itonaga and another referee for their help in evaluating this paper.

\section{References}

Araki, T., Global structure of geomagnetic sudden commencements, Planet. Space Sci., 25, 373-384, 1977.

Araki, T., A physical model of the geomagnetic sudden commencement, in Solar Wind Sources of Magnetispheric Ultra-LowFrequency Waves, Ed M. J. Engebreston, K. Takahashi, and M. Scholer, pp. 183-200, Geophysical Monograph 81, 1994.

Banks, P. M., and G. Kockarts, Aeronomy, Part A, Academic Press, 1973.

Denisenko, V. V., and S. S. Zamay, Electric field in the equatorial ionosphere, Planet. Space Sci., 40, 941-952, 1992.

Fejer, B. G., Equatorial ionospheric electric fields associated with magnetospheric disturbances, in Solar Wind-Magnetosphere Coupling, Ed Y. Kamide and J. A. Slavin, pp. 519-545, Terra Scientific Publishing Company, Tokyo, 1986.

Fejer, B. G., Low latitude electrodynamic plasma drifts: a review, J. Atmos. Solar-Terr. Phys., 53, 677-693, 1991.

Fejer, B. G., The electrodynamics of the low-latitude ionosphere: recent results and future challenges, J. Atmos. Solar-Terr. Phys., 59, 1465-1482, 1997.

Fejer, B. G., and L. Scherliess, Empirical models of storm time equatorial zonal electric fields, J. Geophys. Res., 102, 24 04724 056, 1997.

Fejer, J. A., Semidiurnal currents and electron drifts in the ionosphere, J. Atmos. Terr. Phys., 4, 184-203, 1953.

Forbes, J. M., and R. S. Lindzen, Atmospheric solar tides and their electrodynamic effects - I. The global Sq current system, J. Atmos. Terr. Phys., 38, 897-910, 1976a.

Forbes, J. M., and R. S. Lindzen, Atmospheric solar tides and their electrodynamic effects - II. The equatorial electrojet, J. Atmos. Terr. Phys., 38, 911-920, 1976b.
Fujii, R., and T. Iijima, Control of the ionospheric conductivities on large-scale Birkeland current intensities under geomagnetic quiet conditions, J. Geophys. Res., 92, 4505-4513, 1987.

Fujii, R., T. Iijima, T. A. Potemra, and M. Sugiura, Seasonal dependence of Large-scale Birkeland currents, Geophys. Res. Lett, 8, 1103-1106, 1981.

Harel, M., R. A. Wolf, P. H. Reiff, R. W. Spiro, W. J. Burke, F. J. Rich, and M. Smiddy, Quantitative simulation of a magnetospheric substorm 1. Model logic and overview, J. Geophys. Res., 86, 2217-2241, 1981a.

Harel, M., R. A. Wolf, R. W. Spiro, P. H. Reiff, C.-K. Chen, W. J. Burke, F. J. Rich, and M. Smiddy, Quantitative simulation of a magnetospheric substorm 2. Comparison with observations, J. Geophys. Res., 86, 2242-2260, 1981 b.

Kamide, Y., and S. Matsushita, Simulation studies of ionospheric electric fields and currents in relation to field-aligned currents 1 . Quiet periods, J. Geophys. Res., 84, 4083-4098, 1979a.

Kamide, Y., and S. Matsushita, Simulation studies of ionospheric electric fields and currents in relation to field-aligned currents 2. Substorms, J. Geophys. Res., 84, 4099-4115, 1979 b.

Kikuchi, T., and T. Araki, Horizontal transmission of the polar electric field to the equator, J. Atmos. Terr. Phys., 41, 927-936, 1979.

Kikuchi, T., T. Ishimine, and H. Sugiuchi, Local time distribution of HF Doppler frequency deviations associated with storm sudden commencements, J. Geophys. Res., 90, 4389-4393, 1985.

Kikuchi, T., H. Lühr, T. Kitamura, O. Saka, and K. Schlegel, Direct penetration of the polar electric field to the equator during a DP2 event as detected by the auroral and equatorial magnetometer chains and the EISCAT radar, J. Geophys. Res., 101, 17 161-17 173, 1996.

Maeda, H., T. Iyemori, T. Araki, and T. Kamei, New evidence of meridional current system in the equatorial ionosphere, Geophys. Res. Lett., 9, 337-340, 1982.

Matsushita, S., On geomagnetic sudden commencements, sudden impulses, and storm durations, J. Geophys. Res., 67, 3753-3777, 1962.

Musmann, G., and E. Seiler, Detection of meridional currents in the equatorial ionosphere, J. Geophys., 44, 357-372, 1978.

Nagata, T., S. Kokubun, and T. Iijima, Geomagnetically conjugate relationship of polar geomagnetic disturbances - Particularly the distinct geomagnetic conjugacy between Syowa station in Antarctica and Reykjavik in Iceland, JARE 1956-1962, Sci. Rep., Ser. A, 3, 1966.

Nisbet, J. S., M. J. Miller, and L. A. Carpenter, Currents and electric fields in the ionosphere due to field-aligned auroral currents, J. Geophys. Res., 83, 2647-2657, 1978.

Nishida, A., N. Iwasaki, and T. Nagata, The origin of fluctuations in the equatorial electrojet; a new type of geomagnetic variations, Ann. Geophysicae, 22, 478-484, 1966.

Nopper, R. W., Jr., and R. L. Carovillano, Polar-equatorial coupling during magnetically active periods, Geophys. Res. Lett., 5, 699-702, 1978.

Papamastorakis, I., G. Haerendel, and W. Baumjohann, Local time dependence of the response of the equatorial electrojet to DP2 and SI disturbances, J. Geophys., 54, 213-218, 1984.

Rastogi, R. G., Longitudinal variation of sudden commencement of geomagnetic storm at equatorial stations, J. Geophys. Res., 98, 15 411-15 416, 1993.

Rastogi, R. G., Midday reversal of equatorial ionospheric electric field, Ann. Geophysicae, 15, 1309-1315, 1997.

Rastogi, R. G., and N. S. Sastri, On the occurrence of SSC (-+) at geomagnetic observatories in India, J. Geomag. Geoelectr., 26, 529-537, 1974.

Reddy, C. A., V. V. Somayajulu, and K. S. Viswanathan, Backscatter radar measurements of storm-time electric field changes in the equatorial electrojet, J. Atmos. Terr. Phys., 43, 817-827, 1981.

Reiff, P. H., Models of auroral-zone conductances, in Magnetospheric Currents, Geophys. Monogr. Ser., Vol. 28, Ed T. A. Potemra, pp. 180-191, AGU, Washington, D. C., 1984. 
Richmond, A. D., Equatorial electrojet - I. Development of a model including winds and instabilities, J. Atmos. Terr. Phys., 35, 1083-1103, 1973.

Saito, H., N. Sato, Y. Tonegawa, T. Yoshino, and T. Saemundsson, Seasonal and diurnal dependence of Pc3-5 magnetic pulsation power at geomagnetically conjugate stations in the auroral zones, J. Geophys. Res., 94, 6945-6948, 1989.

Sarma, S. V. S., and T. S. Sastry, On the equatorial electrojet influence on geomagnetic pulsation amplitudes, J. Atmos. Terr. Phys., 57, 749-754, 1995.

Sastri, J. H., J. V. S. V. Rao, and K. B. Ramesh, Penetration of polar electric fields to the nightside dip equator at times of geomagnetic sudden commencements, J. Geophys. Res., 98, 17 517-17 523, 1993.

Sastri, J. H., Y. N. Huang, T. Shibata, and T. Okuzawa, Response of equatorial-low latitude ionosphere to sudden expansion of magnetosphere, Geophys. Res. Lett., 22, 2649-2652, 1995.

Sastri, J. H., M. A. Abdu, and J. H. A. Sobral, Response of equatorial ionosphere to episodes of asymmetric ring current activity, Ann. Geophysicae, 15, 1316-1323, 1997.

Senior, C., and M. Blanc, On the control of magnetospheric convection by the spatial distribution of ionospheric conductivities, J. Geophys. Res., 89, 261-284, 1984.

Spiro, R. W., R. A. Wolf, and B. G. Fejer, Penetration of highlatitude-electric-field effects to low latitudes during SUNDIAL 1984, Ann. Geophysicae, 6, 39-49, 1988.

Sugiura, M., and J. C. Cain, A model equatorial electrojet, J. Geophys. Res., 71, 1869-1877, 1966.

Takeda, M., Three-dimensional structure of ionospheric currents produced by field-aligned currents, J. Atmos. Terr. Phys., 44, 695-701, 1982.
Tarpley, J. D., The ionospheric wind dynamo - I Lunar tide, Planet. Space Sci., 18, 1075-1090, 1970.

Tsunomura, S., On the conjugate relationships of the amplitudes of SSCs and SIs observed at Reykjavik and Syowa, Mem. Kakioka Mag. Obs., 23, 7-16, 1989.

Tsunomura, S., and T. Araki, Numerical analysis of equatorial enhancement of geomagnetic sudden commencement, Planet. Space Sci., 32, 599-604, 1984.

Untiedt, J., A model of the equatorial electrojet involving meridional currents, J. Geophys. Res., 72, 5799-5810, 1967.

Vickrey, J. F., R. C. Livingston, N. B. Walker, T. A. Potemra, R. A. Heelis, M. C. Kelley, and F. J. Rich, On the current-voltage relationship of the magnetospheric generator at intermediate spatial scales, Geophys. Res. Lett., 13, 495-498, 1986.

Yumoto, K., H. Osaki, K. Fukao, K. Shiokawa, Y. Tanaka, S. I. Solovyev, G. Krymskij, E. F. Vershinin, V. F. Osinin, and $210^{\circ}$ MM Magnetic Observation Group, Correlation of high- and low-latitude $\mathrm{Pi} 2$ magnetic pulsations observed at $210^{\circ}$ magnetic meridian chain stations, J. Geomag. Geoelectr., 46, 925-935, 1994.

Yumoto, K. and the $210^{\circ} \mathrm{MM}$ Magnetic Observation Group, Initial results from the $210^{\circ}$ magnetic meridian project-Review, J. Geomag. Geoelectr., 47, 1197-1213, 1995.

Yumoto, K., H. Matsuoka, H. Osaki, K. Shiokawa, Y. Tanaka, T. Kitamura, H. Tachihara, M. Shinohara, S. I. Solovyev, G. A. Makarov, E. F. Vershinin, A. V. Buzevich, S. L. Manurung, O. Sobari, M. Ruhimat, Sukmadradjat, R. J. Morris, B. J. Fraser, F. W. Menk, K. J. W. Lynn, D. G. Cole, J. A. Kennewell, J. V. Olson, and S.-I. Akasofu, North/south asymmetry of sc/si magnetic variations observed along the $210^{\circ}$ magnetic meridian, J. Geomag. Geoelectr., 48, 1333-1340, 1996. 